\title{
Review of Dementia Reimagined: Building a Life of Joy and Dignity from Beginning to End by Tia Powell
}

\author{
Jack C. Lennon ${ }^{\mathrm{a}, \mathrm{b}, \mathrm{c}, *}$ \\ ${ }^{a}$ Department of Psychology, Adler University, Chicago, IL, USA \\ ${ }^{\mathrm{b}}$ Department of Neurological Sciences, Section of Parkinson's Disease and Movement Disorders, \\ Rush University Medical Center, Chicago, IL, USA \\ ${ }^{\mathrm{c}}$ Department of Behavioral Sciences, Rush NeuroBehavioral Center, Rush University Medical Center, \\ Skokie, IL, USA
}

Accepted 4 October 2019

\begin{abstract}
This review of Dementia Reimagined: Building a Life of Joy and Dignity from Beginning to End by Dr. Tia Powell describes its unique, scientifically-informed approach to the topics of caring for family members with dementia within the confines of the current United States healthcare system and preparing for their long-term care. Her book serves to provide an important account of many difficulties confronted along the course of dementia, ultimately seeking compassionate, dignified care for a loved one during the many years following diagnosis that allow for the experiencing of life's joys that are unique to each individual. This book is tailored to a broad readership, providing information for all individuals caring for people with dementia, and maintains a candid voice as it relates to our pursuit of curative treatments for an incurable illness. This review raises further questions related to how autonomy and beneficence can be amplified when interdisciplinary conflicts arise, as well as how education reform may improve patient care.
\end{abstract}

Keywords: Aging, Alzheimer's disease, dementia, ethics, healthcare

Dr. Tia Powell's Dementia Reimagined: Building a life of Joy and Dignity from Beginning to End [1], published by Penguin Random House in 2019, is an enlightening and unflinchingly honest discussion of dementia, serving as a scientifically- and historically-insightful account of dementia's progression as a broad concept. As a professor of psychiatry and bioethics at Albert Einstein College of Medicine in New York, directing the Montefiore Einstein Center for Bioethics, her work is friendly to a wide readership and illuminates the importance of realis-

*Correspondence to: Jack C. Lennon, MA, 17 N Dearborn St, Chicago, IL 60602, USA. E-mail: jlennon@ adler.edu; ORCID iD: 0000-0002-8490-2807 tic decision-making and preparation of care amidst an illness that, in spite of ongoing efforts, is currently incurable.

Dementia Reimagined is comprised of 12 chapters, beginning with fundamental components of the book's narrative, such as her family's experience with dementia and what this concept entails. The remaining chapters allow for chronological movement across dementia care and knowledge from early decades to present day, such as discussions of 18thcentury Bedlam and the mistreatment of mentally-ill patients, followed by the momentous research of Solomon Carter Fuller and other pioneers in the early 19th century. Powell proceeds to discuss specific 
components of modern dementia care-economic, sociopolitical, interpersonal-that are often misunderstood until the situation presents itself. In many ways, families are ill-prepared for what lies ahead in the care for loved ones with dementia and, thus, must be armed with knowledge. The book continues to weave this care continuum into the ongoing dementia research, explaining her concern for those who may never benefit from these studies but also receive suboptimal care, until care, financial preparation, and proxy decision-making become the central foci.

Powell reports that much of what she has learned over time, through experience and ongoing academic endeavors, is well beyond that which she learned during medical school at Yale University in the 1980s. Her background in the tenets of bioethics-autonomy and beneficence in the management of illness-seems to be a driving force behind her voice and views on dementia. Powell and her five siblings witnessed both their grandmother and mother pass away with dementia after her medical education, with Powell taking an observational position with her grandmother and serving as health care proxy for her mother from both near and far. Powell would face the socioeconomic, ethical, and nuanced decision-making difficulties that she was not taught in medical school, along with the ethical considerations of treating comorbid conditions in the context of an incurable illness that was significantly less understood in the 1980s.

Powell's parents returned to Maryland from Florida following discussions related to the notable cognitive decline of her mother and the need to be closer to Powell's two siblings and medical colleagues who still resided in their hometown. During the course of hospice care, her father passed away with cancer. His home health aide continued to care for Powell's mother following his passing, but her cognitive state would only worsen following the loss of her husband. Memory decline, paranoia, medication management, and reduced driving capacity took a toll for many years until further medical complications required more intensive care. Powell was the health care proxy yet, in the context of heart block or abnormally slow heart rhythm due to blockage of critical electrical signals, discovered that she was not the sole decision-maker. After witnessing her grandmother extend her life with a pacemaker while struggling in other areas of well-being, Powell's mother decided in advance that she would not agree to a pacemaker. Her mother would continue to require hospitalization on various occasions, with each protocol requiring informed consent from a patient cognitively incapable of providing it. Along came the day, however, that Powell met a cardiologist who was focused on the heart block as one, discrete concern rather than one of many, the treatment of which would prolong life but not improve other medical problems. Her mother, without fully understanding the procedure, agreed to a pacemaker only to later recant this decision. She continued to require hospitalizations thereafter until heart disease took her life.

Readers are introduced to the various levels of knowledge healthcare professionals have possessed over time, moving chronologically from the reprehensible treatment of people with dementia to more modern neurobiological findings that have been rigorously investigated over the decades. Almost symbolically, many chapters seem to discuss the collision of scientific progress and hindrance. Powell states on numerous occasions that $99.6 \%$ of experimental Alzheimer's medications result in failure to cure or reverse the condition (p. 129). Even of those that pass safety requirements of Phase $1,98 \%$ fail subsequent efficacy trials. PET scans and other imaging tools have been involved in discoveries that, to Powell, provide limited clinical utility in terms of care and simply create a sense of scientific legitimacy amidst an elusive illness with significant overlap across differentials. With noted oscillations between concepts of origin such as beta-amyloid and tau pathologies, along with the role of $A P O E$ and its polymorphisms, Powell states that a cure will not stem from an overnight breakthrough.

Powell describes in a digestible manner the forces that drive certain forms of treatment and research studies, such that the search for a cure has seemingly presided over more pressing matters. Powell's intellectual approach to deeply personal life circumstances offers realistic optimism of care amidst inconvenient truths related to the terminal nature of dementia and late-stage complications, such as eating and swallowing difficulties. This knowledge is necessary if one is to absorb Powell's message- to prepare in advance for that which is inevitable and improve upon that which is not, such as taking advantage of the cognitive and emotional faculties that are not lost during the early and middle stages of dementia and ensuring that its later stages are met with the individual's unique desires.

Individuals who have developed or will soon develop dementia, including the Baby Boomer gen- 
eration, are unlikely to witness its cure due to a long history of failures and the length of time required for any breakthrough to become accessible. While we can certainly hope and contribute to these causes by participating in research projects, she argues that preparation for all end results is paramount. This preparation includes meaningful discussions with family members regarding their wishes over the many years during which they maintain adequate functioning, how they envision their care, and which types of treatments and life-saving procedures to which they are open. Advance directives do not absolve all burden, rendering these early discussions important for the time at which the individuals with dementia are no longer capable of articulating their desires. Powell offers unique approaches by medical professionals who often phrase the need for interventions in misleading ways. It is noted that these could be avoided by improved education in medicine and palliative care and remaining loyal to autonomy and beneficence.

Powell has provided a rich, chronological history of dementia stigma, classification, research, and its medical underpinnings. Powell's hope, however, is derived from dignified care rather than potential cures. Therefore, her concern for those who are unlikely to be cured outweighs any promise research may offer. Given this focus, a useful addition would have been a thorough evaluation of how medical education could be altered to align with Powell's views while simultaneously remaining faithful to tenets of bioethics across medical specialties. As Powell notes, not all families are well-informed and armed with medical backgrounds and, even when provided with information on all possible treatments, may depend on the physicians' recommendations. One must question the point at which a specialist should determine that a life-saving and -prolonging procedure is unwarranted in the context of an incurable illness like dementia. When timing is critical, how
Powell would like these situations to unfold is an important consideration. A common thread may be ensuring patient dignity but, when faced with treatable conditions and physicians' fears of malpractice, competing, interdisciplinary interests may arise. If a simple solution to this dilemma is nonexistent, then a warranted discussion would pertain to how education and knowledge can be made more accessible for those who are either lacking an understanding of dementia or unequipped to face these complex, medical situations with competing, dissonant emotional responses. A more actionable conclusion may have better served readers of all backgrounds, allowing them to serve as agents of change in the face of all sociopolitical, economic, and ethical obstacles noted by Powell.

Dementia Reimagined [1] is an informative, scholarly work of emotional depth for professionals as well as those without requisite knowledge of dementia. The sequential approach to a complex topic is an optimal manner to incorporate both the science and the emotional components of dementia care. There are lessons to be learned by readers regardless of profession, with this book serving as a practical view of dementia for the sake of human dignity and well-being. Those with dementia need not be victims of its symptoms but, instead, live all remaining moments comfortably and, ideally, as they envisioned and planned for years in advance.

\section{DISCLOSURE STATEMENT}

The author has no conflict of interest to report.

\section{REFERENCES}

[1] Powell T (2019) Dementia reimagined: Building a life of joy and dignity from beginning to end, Penguin Random House LLC, New York. 